\title{
Design of soft x-ray gratings for free electron lasers: from specification to characterization.
}

\author{
Maurizio Vannoni*a , Daniele La Civita ${ }^{\mathrm{a}}$, Rolf Follath ${ }^{\mathrm{b}}$, \\ Liubov Samoylova ${ }^{\mathrm{a}}$, Frank Siewert ${ }^{\mathrm{c}}$, Harald Sinn ${ }^{\mathrm{a}}$ \\ a European XFEL GmbH, Albert-Einstein-Ring 19,22761 Hamburg, Germany \\ ${ }^{\mathrm{b}}$ Swiss Light Source, Paul Scherrer Institute, CH-5232 Villigen-PSI, Switzerland \\ ${ }^{\mathrm{c}}$ Helmholtz Zentrum Berlin, BESSY-II, Institute for Nanometre Optics and Technology, Albert- \\ Einstein-Str. 15, 12489 Berlin, Germany
}

\begin{abstract}
The European XFEL is a large facility under construction in Hamburg, Germany. It will provide a transversally fully coherent X-ray radiation with outstanding characteristics: high repetition rate (up to 2700 pulses with a 0.6 milliseconds long pulse train at $10 \mathrm{~Hz}$ ), short wavelength (down to $0.05 \mathrm{~nm}$ ), short pulse (in the femtoseconds scale) and high average brilliance $\left(1.6 \cdot 10^{25}\right.$ photons $/ \mathrm{s} / \mathrm{mm}^{2} / \mathrm{mrad}^{2} / 0.1 \%$ bandwidth $)$. Due to the very short wavelength and very high pulse energy, mirrors have to present high quality surface, to be very long, and at the same time to implement an effective cooling system. Matching these tight specifications and assessing them with high precision optical measurements is very challenging. One of the three foreseen beamlines operates in the soft X-ray range and it is equipped with a diffractive monochromator. The monochromator is using a variable line spacing grating that covers the wavelength range from 4.6 $\mathrm{nm}$ to $0.41 \mathrm{~nm}$ (energies from $270 \mathrm{eV}$ to $3000 \mathrm{eV}$ ). The grating profile is blazed, and due to the small angle and relatively few lines $/ \mathrm{mm}$, it is also very challenging to realize and to be characterized. In this contribution we discuss about the requirements of the optics involved in the soft X-ray monochromator. We describe mirror and grating specifications, and the tests that could be carried out during and after the manufacturing in order to ensure the specifications match.
\end{abstract}

Keywords: X-Rays Optics, grating, optical testing

\section{INTRODUCTION}

The European $\mathrm{XFEL}^{1}$ is a new Free Electron Laser, currently under construction in Hamburg, with the characteristics outlined in the abstract. The capability that sets the European XFEL facility apart from all other hard X-ray lasers or third generation synchrotron sources is the Megahertz repetition rate of FEL pulses in trains of up to 2700 pulses and the high average brilliance of each pulse. That means up to several 10 Kilowatt heat load per $\mathrm{mm}^{2}$ on some optical elements, for the duration of a pulse train, while the average heat load is comparable or lower than other synchrotron sources. Considering this tremendous heat load, partially transferred to the various optical elements inside the beamlines, local surface deformations are expected and therefore the beam quality will be deteriorated, especially its spatial coherence that is one of the most desired key feature. To solve this issue, additional steady-state and transitory analyses through finite element calculations have been used to predict thermal effects and a proper cooling system will be provided. ${ }^{2}$ However, to minimize the problem, the x-ray beam is also spread out over a large footprint in the grazing incidence reflections, resulting in very long optical elements. The desired optical requirements of these long elements are comparable with the "standard" but shorter ones already under operation in synchrotron facilities: in our case they range from $500 \mathrm{~mm}$ long gratings up to 1 meter long flat mirrors, with required peak-to-valley errors of few nanometers on their full aperture. Reaching this accuracy level becomes challenging on the desired length dimensions. In addition, another problem is to characterize these optical elements to ensure that the required surface quality has been reached and, for the gratings, that the ruling has been properly done. Correct metrology and characterization is also a challenging task: using a deterministic polishing to achieve the best surface, the manufacturing and characterization processes are connected together and an incorrect metrology could result in an out of specifications optical element.

*maurizio.vannoni@xfel.eu; phone +49 040 89985456; fax +49 040 89981905; www.xfel.eu

Modeling Aspects in Optical Metrology IV, edited by Bernd Bodermann, Karsten Frenner, Richard M. Silver, Proc. of SPIE Vol. 8789, 878916 - @ 2013 SPIE · CCC code: 0277-786X/13/\$18 · doi: 10.1117/12.2020520 
Among the different European XFEL beamlines, we are now considering just the soft x-rays one, called SASE3 beamline. Three different energies will be provided for the electron beam injected in this beamline: $10.5 \mathrm{GeV}, 14.0 \mathrm{GeV}$ and $17.5 \mathrm{GeV}$. Produced x-rays energies will span from $4.6 \mathrm{~nm}(=270 \mathrm{eV})$ to $0.41 \mathrm{~nm}(=3 \mathrm{keV})$, with a single pulse duration ranging from 2 to 100 femtoseconds. The typical spectral bandwidth expected ${ }^{1}$ is $5 \cdot 10^{-3}$, and for some particular applications it is required to reduce the bandwidth even more. For that reason a monochromator have to be inserted on the beam path, in order to have a spectral bandwidth up to $5 \cdot 10^{-5}$, depending on the particular wavelength used and the alignment conditions. The expected pulse length will be stretched up to 100 femtoseconds, but it will be still short enough to allow a high repetition rate and time-resolved experiments. The monochromator can also be bypassed: moving out of the beam path the monochromator first optical element allows the unfiltered beam ("pink beam") to go directly to the experimental hall. A schematic view of the beam transport optical setup is depicted in Fig. 1.

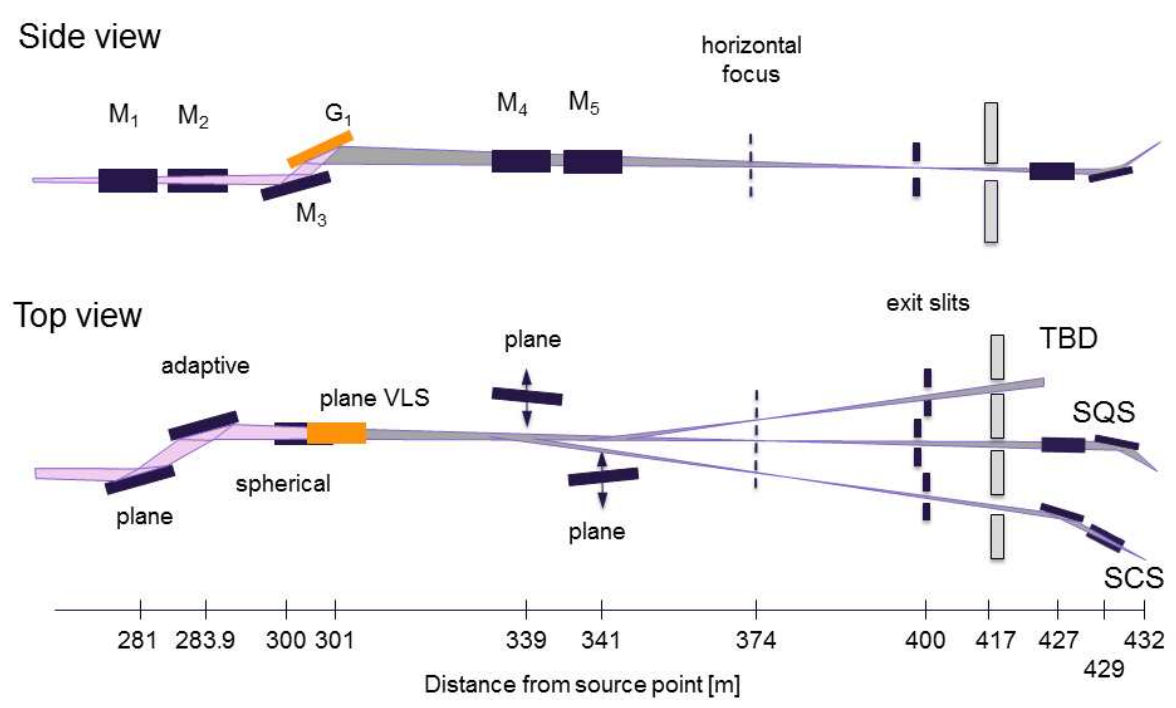

Figure 1. European XFEL SASE3 beamline: optical setup. $\mathrm{M}_{1}$ and $\mathrm{M}_{2}$ are two flat offset mirrors mainly used for radiation safety reasons. The first has fix shape, whereas the second has the possibility to change the surface through an adaptive piezoelectric system. $M_{3}$ is a focusing mirror; $G_{1}$ is the monochromator grating while $M_{4}$ and $M_{5}$ are used to distribute the beam to the selected experimental station.

In this setup every optical element is challenging, in particular the monochromator grating and the associated mirrors. In order to ensure the correct setup of the system and to achieve the desired x-rays beam characteristics, a careful engineering of each part is needed and a correct metrology approach must be provided. We will focus in particular on the grating element of the monochromator and on the linked $\mathrm{M}_{3}$ mirror. Two exchangeable mirrors and two gratings with line densities of 50 lines $/ \mathrm{mm}$ and 150 lines $/ \mathrm{mm}$, to cover the full energy range, will be provided. In the next sections, we will show the design and specifications of these gratings and mirrors, together with the required characterization.

\section{MONOCHROMATOR DESIGN AND SPECIFICATIONS}

The SASE3 soft x-ray monochromator, as depicted in Fig. 1, employs a Variable Line Spacing Plane Grating Monochromator (VLS-PGM) with the plane grating positioned in a converging beam. In the vertical (dispersive) plane, the focus distance is determined by the spherical mirror $\mathrm{M}_{3}$ and the magnification ratio of the grating. In the horizontal plane, the focus position is determined by the adaptive $\mathrm{M}_{2}$ mirror while the plane mirrors $\mathrm{M}_{4}$ and $\mathrm{M}_{5}$ are used to distribute the beam to three different experimental stations. To keep the beamline in focus for all photon energies, two different mirrors $\mathrm{M}_{3}$ are used alternatively.

In the low photon energy range $(270 \mathrm{eV}-1.2 \mathrm{keV})$, a spherical pre-mirror $\mathrm{M}_{3} \mathrm{~B}$ with a radius of curvature of 7.5 $\mathrm{km}$ and a grazing incidence angle of 20 milliradians is used. In the high photon energy range $(1.2 \mathrm{keV}$ to $3 \mathrm{keV})$ a premirror $\mathrm{M}_{3} \mathrm{~A}$ with a radius of curvature of $16.7 \mathrm{~km}$ and a grazing incidence angle of 9 milliradians is instead used. Both gratings can be operated with each mirror. The grating $\mathrm{G}_{1}$, with a line density of 50 lines $/ \mathrm{mm}$ and a nominal resolution up to 14700 , is intended for low spectral resolution experiments with a ultrashort pulse length. The grating $\mathrm{G}_{2}$, with line density 150 lines $/ \mathrm{mm}$, is more suitable for high spectral resolution applications and still a not so long pulse length. In 
any case, the pulse length stays below 100 femtoseconds. Both gratings will have blazed profiles. The blazed profile has been chosen to enhance the diffraction efficiency and to have higher damaging threshold levels. It has been shown that laminar profiles are more sensitive to high power beams and therefore more susceptible to beam damage ${ }^{3}$. The premirrors have fixed positions and grazing incidence angles, to keep the setup simple, while the two gratings are interchangeable and can be rotated to select the photon energy.

The gratings have been designed to keep the distance to the exit slit constant at $l=99 \mathrm{~m}$. This can be accomplished by using a variable line separation $d(x)$ according to

$$
d(x)=d_{0}\left(1+v_{1} x+v_{2} x^{2}+\cdots\right) .
$$

where $d_{0}$ is the central line spacing and $v_{1}$ and $v_{2}$ are free parameters. From the literature ${ }^{4}$ we know that $v_{1}$ parameter can be calculated as

$$
v_{1}=\frac{d_{0}}{n \lambda l}\left(\cos ^{2} \alpha-\cos ^{2} \beta\right)
$$

where $n$ is the diffraction order number ( $=1$ in our case), $\lambda$ is a selected wavelength, $\alpha$ and $\beta$ are respectively the incidence and diffraction angle respect to the normal of the grating. The equation can be approximated as

$$
v_{1} \cong \frac{2 d_{0}}{n \lambda l}(\sin \alpha-\sin \beta)
$$

being $\alpha$ and $\beta$ very near to $\pi / 2$. From the grating equation we have

$$
\frac{n \lambda}{d_{0}}=\sin \alpha-\sin \beta
$$

so, combining Eq. 3 and Eq. 4, we have

$$
v_{1} \cong \frac{2}{l}
$$

that in our case means $v_{1} \cong 0.02020 \mathrm{~m}^{-1}$. Using Eq. 1, this parameter creates a maximum line displacement of $0.5 \%$, corresponding to 0.1 micron for the $50 \mathrm{l} / \mathrm{mm}$ grating and 0.03 micron for the $150 \mathrm{l} / \mathrm{mm}$ grating, measured from the center to the edge. Further calculations ${ }^{5}$ give a $v_{2}$ estimation of $1 \cdot 10^{-4} \mathrm{~m}^{-2}$ : in this case we have a negligible effect, about $6 \cdot 10^{-4} \%$, so we can neglect it safely.

The surface quality of both the pre-mirrors and the gratings substrates must be really good, to avoid any wavefront deteriorations that would spoil the properties of the beam at the experiment. To have a comparison, standard optical elements are generally used with visible light and normal angle incidence, and in that case a "standard quality" optical surface has normally a Peak-to-Valley maximum deviation from ideal surface that is around $\lambda / 4$, a "good quality" surface is around $\lambda / 10$ and a "very good quality" surface is better than $\lambda / 20$. In our case, the $\lambda$ parameter is really small, but the incidence angle is also very far from the normal: for a given deviation $\Delta h$ and a grazing incidence, the wavefront distortion $\Delta z$ can be roughly calculated with the following formula: ${ }^{6}$

$$
\Delta z \cong 2 \Delta h \sin \theta
$$

As an order of magnitude, having a $\theta=20 \mathrm{mrad}$ of grazing incidence angle and a Peak-to-Valley deviation of $3 \mathrm{~nm}$, we calculate a wavefront aberration of $\Delta \mathrm{z}=2 \Delta \mathrm{h} \sin \theta=0.12 \mathrm{~nm}$ : if we consider $\lambda=1 \mathrm{~nm}$, this situation is comparable to an optical mirror with $\lambda / 20$ quality. Following these guidelines, the final specifications have been decided and they are reported in Tab.1. With these specifications, full wavefront simulation have been carried out and the calculated beam quality is, in the worst case, better than $\lambda / 8$, and in some cases it is better than $\lambda / 20$. 


\begin{tabular}{|c|c|c|c|c|}
\hline & $\begin{array}{l}\text { M3a pre- } \\
\text { mirror }\end{array}$ & $\begin{array}{l}\text { M3b pre- } \\
\text { mirror }\end{array}$ & G1 grating & G2 grating \\
\hline $\begin{array}{l}\text { Substrate size } \\
(\mathrm{LxWxH}) \mathrm{mm}^{3}\end{array}$ & $600 \times 100 \times 70$ & $600 \times 100 \times 70$ & $530 \times 100 \times 70$ & $530 \times 100 \times 70$ \\
\hline $\begin{array}{l}\text { Optical surface } \\
(\mathrm{LxW}) \mathrm{mm}^{2}\end{array}$ & $580 \times 25$ & $580 \times 30$ & $500 \times 30$ & $500 \times 30$ \\
\hline Figure & Spherical & Spherical & Flat & Flat \\
\hline $\begin{array}{l}\text { Incidence angle } \\
\text { (milliradians) }\end{array}$ & 9 (fixed) & 20 (fixed) & $\begin{array}{c}\max .19 .1(1.5 \mathrm{keV}) \\
\min .5 .7(1 \mathrm{keV})\end{array}$ & $\begin{array}{c}\max .17(1.5 \mathrm{keV}) \\
\min .1 .8(1 \mathrm{keV})\end{array}$ \\
\hline $\begin{array}{l}\text { Radius of curvature } \\
\text { (meters) }\end{array}$ & 16700 & 7480 & $>300.000$ & $>300.000$ \\
\hline Line density & & & $501 / \mathrm{mm}$ & $1501 / \mathrm{mm}$ \\
\hline VLS parameter & & & $2.0202 \mathrm{e}-51 / \mathrm{mm}$ & $2.0202 \mathrm{e}-51 / \mathrm{mm}$ \\
\hline $\begin{array}{l}\text { Groove profile } \\
\text { Angle }\end{array}$ & & & $\begin{array}{c}\text { Blazed } \\
0.1^{\circ}\end{array}$ & $\begin{array}{c}\text { Blazed } \\
0.3^{\circ}\end{array}$ \\
\hline $\begin{array}{l}\text { RMS slope error } \\
\text { (nanoradians) }\end{array}$ & 50 & 50 & 50 & 50 \\
\hline $\begin{array}{l}\text { Residual height error } \\
\text { (P-V) (nanometers) }\end{array}$ & 3 & 3 & 3 & 3 \\
\hline
\end{tabular}

Table 1. VLS-plane monochromator optical components specifications.

\section{OPTICAL ELEMENTS CHARACTERIZATION}

As reported in Tab. 1, designed specifications for monochromator optical elements are challenging and difficult to achieve even with state-of-the-art technology. A proper characterization must be planned and carried out. Now we will examine the main characteristics of the pre-mirrors and gratings, working out the way to perform characterization measurements for every parameter, with particular attention to the final accuracy. When possible, we will consider different methods to measure the same characteristic, examining the different information that we could gather from each one.

\subsection{Pre-Mirror and Gratings surface figure}

Two different methods are generally used to measure the optical surfaces figure: interferometry and deflectometry. Each one has its advantages and disadvantages. Interferometry is used mostly by optics manufacturers and few synchrotron laboratories, ${ }^{7}$ while most synchrotron laboratories have slope measuring profiler like the Long Trace Profiler (LTP) or second generation slope measuring profiler like the Nanometer Optical Component Measuring Machine (NOM) ${ }^{8}$, to test the long spatial frequency contribution to the quality of optical elements.

For interferometry, a large aperture Fizeau instrument can be used, ${ }^{9,10}$ but usually the maximum measurable diameter will be limited by the interferometer beam expander. To overcome this limitation, a grazing incidence setup ${ }^{11}$ or a stitching method ${ }^{12}$ can be used. Advantages of interferometric methods are: data acquisition speed, one-shot measurement in all the field of view, and usage of a standard instrument. Disadvantages for the grazing incidence setup are a reduced sampling interval and smaller height accuracy. Using a stitching method through a translation stage, the accuracy and sampling interval are maintained, but some "stitching errors" can be found between sub-apertures connections due to the mathematical algorithm and translation stage errors. In both the methods we still have to calibrate 
the reference surface attached on the interferometer with an accuracy comparable or even better than desired results accuracy. Expected performances for a Fizeau interferometer setup with $300 \mathrm{~mm}$ beam-aperture diameter are reported in Tab. 2. Basically, a Large Beam Aperture Fizeau interferometer is equipped with a $1 \mathrm{Kx} 1 \mathrm{~K}$ pixels CCD camera, and the accuracy is on a nanometric level if an absolute calibration is carried out, using three-flat test methods ${ }^{13-15}$.

For slope measuring deflectometry, typical instruments use a laser beam reflected over the test mirror surface: depending on the local topography of the surface, the beam is reflected in a different way on a position sensitive detector inside the instrument sensor head. ${ }^{16}$ This position measurement is directly related to the local slope on the mirror surface. In contrast to interferometry this method does not rely on external references, but it is linked to its internal construction. It is noted here that in case of ultra-flat substrates, as required for the European XFEL, the measuring beam of slope measuring sensor will be follow the same path constantly. Thus typical error sources for slope measuring deflectometry like optical inhomogeneity of the FT-lens-glass will not have a significant impact on the error-budget of the instrument. One of these instruments is the NOM: ${ }^{17-19}$ it has been already used extensively to characterize other synchrotron optical elements and, by averaging a large number of scans, the random error budget can be minimized to values below 50 nanoradians (rms). In case of flat surfaces, we have seen that it can be also better that that.

The performances details are also reported in Tab. 2 .

\begin{tabular}{|c|c|c|}
\hline & 300mm Fizeau & NOM \\
\hline $\begin{array}{c}\text { Test mirror } \\
\text { measurable size }\end{array}$ & $\begin{array}{l}300 \mathrm{~mm} \\
\text { diameter }\end{array}$ & $1200 \times 300 \mathrm{~mm}^{2}$ \\
\hline $\begin{array}{l}\text { Angle accuracy on } \\
1 \mathrm{~mm} \text { sample length }\end{array}$ & $\begin{array}{c}150 \\
\text { nanoradians }\end{array}$ & $\begin{array}{c}<50 \\
\text { nanoradians }\end{array}$ \\
\hline $\begin{array}{c}\text { Maximum } \\
\text { measurable angle }\end{array}$ & $\begin{array}{c}263 \\
\text { microradians }\end{array}$ & $\begin{array}{l} \pm 6.6 \\
\text { milliradians }\end{array}$ \\
\hline $\begin{array}{c}\text { Minimum } \\
\text { measurable radius } \\
\text { of curvature }\end{array}$ & $\begin{array}{l}1 \mathrm{~km} \text { (without } \\
\text { additional } \\
\text { optics) }\end{array}$ & $\begin{array}{c}0.9 \text { meter for } \\
\text { NOM-LTP } \\
3.0 \text { meter for } \\
\text { NOM-AC }\end{array}$ \\
\hline Height accuracy & $\begin{array}{l}1 \mathrm{~nm} \text { (absolute } \\
\text { calibration) }\end{array}$ & $1 \mathrm{~nm}$ \\
\hline $\begin{array}{l}\text { Time for a } \\
\text { measurement }\end{array}$ & $\begin{array}{l}302 \\
\text { milliseconds }\end{array}$ & half an hour \\
\hline
\end{tabular}

Table 2. Comparison between two different methods to measure surface figure.

Analyzing the surface specification for pre-mirrors and grating substrates, we can see that the desired maximum slope error (Tab. 1) is in the same range of NOM accuracy. Even the maximum residual height error should be measurable within the Fizeau interferometer specifications. Basically, the two approaches are quite complementary. With deflectometry, we measure the slope error profile and the height profile is derived with numerical integrations; with interferometry, we measure the height profile and we calculate the slope profile with numerical derivation.

We analyze now the maximum radius of curvature admitted for these optical elements. Considering perfectly spherical profiles, the radius of curvature $R$ and the maximum out-of-plane distance $h$ are linked with optics diameter $D$ through the formula:

$$
h=\frac{D^{2}}{8 R}
$$

while the maximum slope angle $\propto$ is

$$
\tan \propto=\frac{D / 2}{R-h}
$$


From these formulas, we calculate a maximum $h=5.62$ microns for the M3b pre-mirror, corresponding to a maximum slope error of $\propto=38.8$ microradians, and a minimum $h=104 \mathrm{~nm}$ (corresponding $\propto=833$ nanoradians) for the gratings. However, with normal incidence interferometry only a limited portion of the mirror is observed at the same time, so for a $300 \mathrm{~mm}$ beam diameter Fizeau system the above value is scaled to $h=1.5$ microns maximum and $h=37.5 \mathrm{~nm}$, minimum.

\subsection{Surface roughness and Power Spectral Density}

The roughness of optical surfaces can be measured with a white-light interferometric microscope. ${ }^{20}$ These instruments use an interferometric setup within their microscope objectives and they have typically sub-nanometer accuracy, but again the main limitation is the influence of the reference surface, that it is included inside the microscope objective. The output is a bi-dimensional profile, and the root-mean-square (rms) roughness value can be easily calculated. Following Ref. 21, the rms roughness of the test $\left(\sigma_{\text {test }}\right)$ and of the reference surface $\left(\sigma_{\text {ref }}\right)$ are connected with the measured profile with the formula:

$$
\sigma_{\text {meas }}^{2}=\sigma_{\text {test }}^{2}+\sigma^{2}{ }_{r e f}
$$

Two methods can be used to achieve $0.1 \mathrm{~nm}$ accuracy for the roughness measurement. We can perform two measurements in two different parts of the sample, then we calculate the difference profile between them (Diff) to cancel the reference influence, and the result is processed with a scaling factor of $\sqrt{2}$ :

$$
\sigma_{\text {test }}=\frac{1}{\sqrt{2}} \sigma_{\text {Diff }}
$$

Another method is to use an almost perfectly polished optical surface, doing several measurements in different parts of this surface, and then averaging the results: the resulting profile is an estimation of the reference profile inside the interferometric microscope objective, and it can be removed from any other further measurement. The estimated reference rms roughness is connected to the "real" one, depending from the surface quality of the mirror used and the number of measurements $N$ :

$$
\sigma_{\text {estimated }}=\sqrt{\left(\frac{\sigma_{\text {mirror }}}{\sqrt{N}}\right)^{2}+\sigma_{\text {reference }}}
$$

With a surface correction, other kind of calculation could be used, as Power Spectra Density estimations that are generally used to provide a full simulation of the x-ray beam after the monochromator. Typical height resolution for these instruments is $0.1 \mathrm{~nm}$ while lateral resolution is ranging from 0.64 to 11.8 microns, depending on the objective used.

\subsection{Grating variable line spacing parameters}

As we have seen (Tab. 1), the variable line spacing parameter required along the grating is quite small. The method proposed to characterize it, is to measure the profile surface in a Littrow setup. ${ }^{22,23}$ This method was chross-checked in the past and have shown excellent agreement with results of groove density measurements achieved by Carl-Zeiss. ${ }^{24}$ In this setup, the incidence and diffraction angle are coincident. Using Eq. 4:

$$
\frac{n \lambda}{2 d}=\sin \alpha
$$


where $d$ is given by Eq. 1 . If $\lambda=632.8 \mathrm{~nm}$ is used to measure the grating, this angle is 0.91 degrees for the 50 lines $/ \mathrm{mm}$ and 2.72 degrees for the 150 lines $/ \mathrm{mm}$. The expected maximum variations for these angles along the grating, are \pm 80 microradians and \pm 240 microradians, respectively.
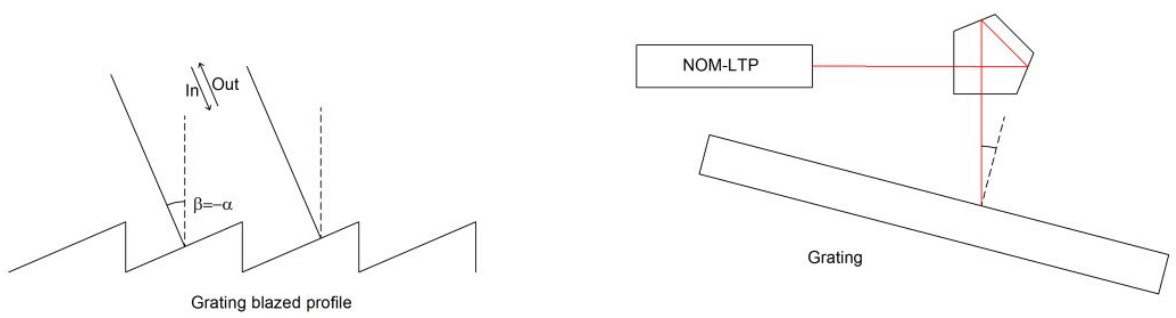

Figure 2. Inspection of groove density under Littrow-condition by use of a Long Trace Profiler.

\subsection{Grating blaze angle}

The blaze-angle of a grating can be inspected by different methods, depending on the grating resolution. Atomic Force Microscope (AFM) is a standard instrumentation at the HZB-Grating and Optics Laboratory and it is used to monitor the manufacturing process. Especially with high-density gratings, AFM can take advantage of a nanometer-resolution in the three axes, and the contact-mode approach can measure even the blaze profile edges. Another method, White-light interferometric profilometry (WLI), can be used properly with low-density gratings: using a 50x microscope objective we can reach a lateral resolution of 0.64 microns and a field of view of $140 \times 110$ microns $^{2}$. In both gratings, the maximum height for the blazed profile is designed to be $35 \mathrm{~nm}$, with a tolerance of few nanometers. To have a reliable measurement of this variable, a calibration with a comparison with known steps of the same order of magnitude should be carried out.

A final test will be performed by testing the grating performance with synchrotron light, with defined energies and a reflectometer, at the BESSY-II-optics beamline ${ }^{25}$. From these measurements, the blaze angle of the grating is reconstructed with high precision.

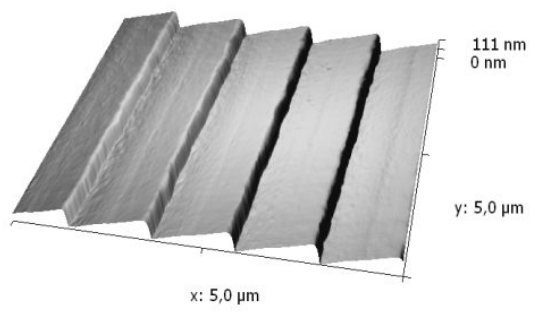

Figure 3. Blazed-grating (1000 lines/mm) after mechanical ruling, measured with an AFM.

\section{CONCLUSIONS}

The specifications of the European XFEL SASE3 monochromator optical elements have been showed and some basic considerations about the necessary characterization measurements have been carried out. From the analysis, the requirements result really challenging for present measurements technology. However, we have presented some methods, deflectometry and interferometry, WLI and AFM, with performances near to the accuracy requested. The approaches are quite complementary, starting from a slope error measurement in the case of deflectometry, while measuring the height error in the case of interferometry, and using contact or non-contact in the case of AFM and WLI. 
Prototypes manufacturing is on the way and preliminary measurements will be carried out in the next future following the presented guidelines.

\section{REFERENCES}

[1] M. Altarelli, et al. (Eds.) XFEL, “The European X-ray Free-Electron Laser”, Technical Design Report, (2006), DESY 2006-097

[2] H. Sinn, M. Dommach, X. Dong, D. La Civita, L. Samoylova, R. Villanueva, and F. Yang, "X Ray Optics and Beam Transport”, Technical Design Report, (2012), XFEL.EU TR-2012-006

[3] J. Gaudin et al., "Investigating the interaction of x-ray free electron laser radiation with grating structure", Opt. Lett. 37, 3033-3035 (2012)

[4] M. Hettrick and S. Bowyer, "Variable line-space gratings: new designs for use in grazing incidence spectrometers," Appl. Opt. 22, 3921-3932 (1983).

[5] L. Poletto, G. Naletto, and G. Tondello, "Grazing-incidence flat-field spectrometer for high-order harmonic diagnostics," Opt. Eng. 40, 178-185 (2001)

[6] L. Samoylova, H. Sinn, F. Siewert, H. Mimura, K. Yamauchi, et al. "Requirements on hard x-ray grazing incidence optics for European XFEL: analysis and simulation of wavefront transformations", Proc. SPIE 7360, EUV and X-Ray Optics: Synergy between Laboratory and Space, 73600E (2009)

[7] Ludbrook G.D., Alcock S.G., Scott S., "A double-pass Fizeau interferometer system for measuring the figure error of large synchrotron optics.” Proc. SPIE 7801 (2010).

[8] Frank Siewert, Tino Noll, Thomas Schlegel, Thomas Zeschke, and Heiner Lammert, "The Nanometer Optical Component Measuring machine: a new Sub-nm Topography Measuring Device for X-ray Optics at BESSY", AIP Conference Proceedings 705, American Institute of Physics, Mellville, NY, 2004, pp. 847-850

[9] L. Rayleigh, "Interference bands and their application," Nature 48, 212-214 (1893)

[10]R. Brünnagel, H.-A. Oehring, and K. Steiner, "Fizeau interferometer for measuring the flatness of optical surfaces," Appl. Opt. 7, 331-335 (1967)

[11]P. Hariharan, "Interferometric testing of optical surfaces: absolute measurement of flatness", Optical Engineering Vol. 36(9), pp. 2478-2481 (1997)

[12] G. Forbes, P. Murphy, and J. Fleig, "Stitching subaperture data for testing aspheric surfaces," in Frontiers in Optics, OSA Technical Digest (CD) (Optical Society of America, 2004), paper OTuD5.

[13] M. Vannoni and G. Molesini, "Iterative algorithm for three flat test," Opt. Express 15, 6809-6816 (2007)

[14]M. Vannoni and G. Molesini, "Absolute planarity with three-flat test: an iterative approach with Zernike polynomials," Opt. Express 16, 340-354 (2008)

[15] M. Vannoni and G. Molesini, "Three-flat test with plates in horizontal posture," Appl. Opt. 47, 2133-2145 (2008)

[16] R. D. Geckeler, "Optimal use of pentaprism in highly accurate deflectometric scanning," Meas. Sci. Technol. 18, 115-125 (2007)

[17]F. Siewert, J. Buchheim, T. Zeschke, G. Brenner, S. Kapitzki, and K. Tiedtke, "Sub-nm accuracy metrology for ultra-precise reflective X-ray optics,” Nucl. Instrum. Methods Phys. Rev. A635, 552-557 (2010).

[18]F. Siewert, J. Buchheim, and T. Zeschke, "Characterization and calibration of 2nd generation slope measuring profiler," Nucl. Instrum. Methods Phys. Rev. A616, 119-127 (2010)

[19] Frank Siewert, Jana Buchheim, Sébastien Boutet, Garth J. Williams, Paul A. Montanez, Jacek Krzywinski, and Riccardo Signorato, "Ultra-precise characterization of LCLS hard X-ray focusing mirrors by high resolution slope measuring deflectometry," Opt. Express 20, 4525-4536 (2012)

[20] Paul J. Caber, "Interferometric profiler for rough surfaces," Appl. Opt. 32, 3438-3441 (1993)

[21] Creath, K. and Wyant, J. C., "Absolute measurement of surface roughness," Appl. Opt. 29, 3823-3827 (1990).

[22] S.C. Irick and W.R. McKinney, "Measurement of Diffraction Gratings with a Long Trace Profiler with Application for Synchrotron Beamline Gratings", Synchrotron Radiation Instrumentation, $10^{\text {th }}$ U.S. National Conference, AIP Proceedings 417, (1997)

[23] D. Cocco, G. Sostero, and M. Zangrando, "Technique for measuring the groove density of diffraction gratings using the long trace profiler," Rev. Sci. Instrum. 74, 3544 (2003) 
[24] Frank Siewert, Heiner Lammert, Gerd Reichardt, Ulrich Hahn, Rolf Treusch, Ruben Reininger, "Inspection of a Spherical Triple VLS-Grating for Self-Seeding of FLASH at DESY," AIP Conference Proceedings 879, 667670 (2007)

[25]F. Eggenstein, F. Schäfers, A. Erko, R. Follath, A. Gaupp, B. Löchel, F. Senf, T. Zeschke, “A reflectometer for at-wavelength characterization of gratings," Nucl. Instrum. Methods Phys. Rev., in press 